\title{
RNA-interference-mediated downregulation of Pin1 suppresses tumorigenicity of malignant melanoma A375 cells
}

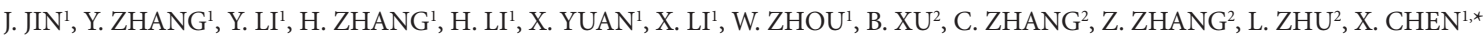 \\ ${ }^{1}$ Department of Pharmacology, Institute of Materia Medica, Chinese Academy of Medical Sciences and Peking Union Medical College, 1 Xian \\ Nong Tan Street, Beijing 100050, PR China; ${ }^{2}$ Department of Medicinal Chemistry, Institute of Materia Medica, Chinese Academy of Medical \\ Sciences and Peking Union Medical College, 1 Xian Nong Tan Street, Beijing 100050, PR China
}

*Correspondence: chenxg@imm.ac.cn, rebeccagold@imm.ac.cn

Received June 15, 2012 / Accepted August 5, 2012

\begin{abstract}
The peptidyl-prolyl isomerase Pin1 is overexpressed in many human cancers, including melanoma. To investigate its possible role in oncogenesis of melanoma and as a therapeutic target, we suppressed Pin1 expression in the human melanoma cell line A375 by microRNA (miRNA) interference technology. Two stable clones with suppressed Pin 1 were established by stable transfection of miRNA plasmid targeting Pin1 into A375 cells. Both clones showed reduced proliferation and invasion in vitro and suppressed tumorigenic potential in athymic mice. Furthermore, Pin1 inhibition also resulted in decreased phosphorylation of Akt and repressed expression of C-Jun N-terminal kinase and pro-matrix metalloproteinase 2, which were associated closely with the development of melanoma. These findings indicate that Pin1 plays an important role in the tumorigenesis of melanoma and might serve as a promising therapeutic target.
\end{abstract}

Key words: malignant melanoma, A375, Pin1, RNA interference, cancer therapy

Malignant melanoma is a cancer of melanocytes, and is the most serious form of skin cancer. Its incidence has been rising steadily throughout the past few decades.[1,2] It is considered curable when detected at an early stage, but once it has entered the advanced stage, it disseminates widely and soon becomes an incurable malignancy with extremely poor prognosis. [3] The median survival time for patients with malignant melanoma is $<1$ year and the 5-year survival rate after initial presentation is $<5 \%$. [4] As a result of the special characteristics and usual resistance to standard chemotherapy, there is no systemic and effective therapy that has a clear effect on overall survival of patients with malignant melanoma. Although our understanding of the molecular biology of malignant melanoma has increased in recent years, detailed knowledge of the molecular mechanisms involved in its formation and progression remains elusive. Furthermore, although after many decades of progress in treating malignant melanoma, many promising new targets are entering the clinic, only a small percentage of patients get benefit from these targeted drugs. $[5,6]$ Therefore, a penetrating understanding of the pathogenesis of malignant melanoma and search for potent molecular targets based on its etiology might lead to effective strategies for the treatment of this cancer.
Oncogenesis is a complex multistep and multifactorial process that ultimately results in uncontrolled cell proliferation and transformation. One of the most important signaling mechanisms involved in this event is phosphorylation of proteins on serine or threonine residues that precede proline (Ser/Thr-Pro).[7] The peptidyl-prolyl isomerase Pin1, which specifically recognizes phosphorylated Ser/Thr-Pro motifs, is an enzyme that promotes the cis-trans isomerization of the peptide bond of its target proteins. These conformational changes can have profound effects on Pin 1 substrates, such as modulating their activity and stability. $[8,9]$ Therefore, Pin 1 has been demonstrated to be involved in the regulation of diverse cellular events, especially proliferation and transformation. A number of studies have suggested that Pin 1 plays an important role in tumorigenesis and is overexpressed in most types of human cancer.[10-15] Furthermore, increased levels of Pin 1 have been shown to correlate with poor clinical outcome, which indicates that the expression level of Pin1 could have become a prognostic marker for diseases such as prostate cancer.[16-18]

Bao et al.[10] have shown that Pin 1 is overexpressed in melanoma. However, it is not known whether Pin 1 is involved 
in the oncogenesis of melanoma, and it is unclear whether Pin1 inhibition affects cell growth or blocks development of melanoma. In the present study, we suppressed the expression of Pin1 in human malignant melanoma A375 cells by microRNA interference (miRNAi) to explore the role and mechanism of Pin 1 in the progression of malignant melanoma.

\section{Materials and methods}

Cell culture. Human malignant melanoma cell line A375, preserved by our laboratory, was cultured in RPMI1640 medium supplemented with $10 \%$ fetal calf serum (Gibco BRL Life Technologies, Grand Island, NY, USA), and incubated at $37^{\circ} \mathrm{C}$ in a humidified atmosphere that contained $5 \% \mathrm{CO}_{2}$.

Pin 1 miRNA plasmids and cell transfection. Four pcDNA6.2-GW/EmGFP-miR-Pin 1 plasmids and the pcDNA6.2-GW/EmGFP-miR-Negative control plasmid were constructed and provided by Invitrogen (Grand Island, NY, USA). The sequences of the four synthesized miRNA oligonucleotides that targeted Pin1 (NM_006221) cloned into the vector, pcDNA6.2-GW/EmGFP-miR, were miRNA1(sense sequence: 5'-TGCTGCTGCCGG TCTGGCTCTTCCTCGTTTTGGCCACTGACTGACGAGGAAGACAGACCGGC AG-3'; antisense: 5'-CCTGCTGCCGGTCTGTCTTCCTCGTCAGTCAGTGGCCA AAACGAGGAAGAGCCAGA CCGGCAGC-3'), miRNA2 (sense sequence: 5'- TGCTGTAGAGGAAGTCGATGTACCTGGTTTTGGCCACTGACTG ACCAGGTACAGACT TCCTCTA-3'; antisense: 5'-CCTGTAGAGGAAGTCTGTAC CTGGTCAGTCAGTGGCCAAAACCAGGTACATCGACTTCCTCTAC-3'), miRNA3 (sense sequence: 5'-TGCTGCATAGCTGCAGCTTGCCATCTGTTTTG GCCACTGACTGACAGATGGC ACTGCAGCTATG-3'; antisense: 5'-CCTGCATA GCTGCAGTGCCATCTGTCAGTCAGTGGCCAAAACAGATGGCAAGCTGCAGCTATGC-3'), miRNA4 (sense sequence: 5'-TGCTGAG ACCGAGAAGGCGTAGCTGA G T T T T G GC CA C T GA C T GA C T CA GCTAC C TTCTCGGTCT-3'; antisense: 5'-CCTGAGACCGAGAAGG TAGCTGAGTCA GTCAGTGGCCAAAACTCAG CTACGCCTTCTC GGTCTC-3'). The sequence of the negative control oligonucleotides was (sense sequence: 5'-TGCTGAAAT GTACTGCGCGTGGAGACGTTTTGGCCACTGACTGACGTCTCCACGCAGT ACATTT-3'; antisense: 5'-CCTGAAATGTACTGCGTGGAGACGTCAGTCAGT GGCCAAAACGTCTCCACGCGCAGTACATTTC-3').

A375 cells were transiently transfected with pcDNA6.2-GW/ EmGFP-miR-Pin 1 plasmids and the pcDNA6.2-GW/EmGFPmiR-Negative control plasmid using Lipofectamine 2000 (Invitrogen) according to the manufacturer's protocol. After $72 \mathrm{hr}$, protein extracts were prepared to select the silencing efficacy of these plasmids. Then, A375 cells were stably transfected with miRNA4, which had the greatest efficiency for suppressing expression of Pin1, and the negative control
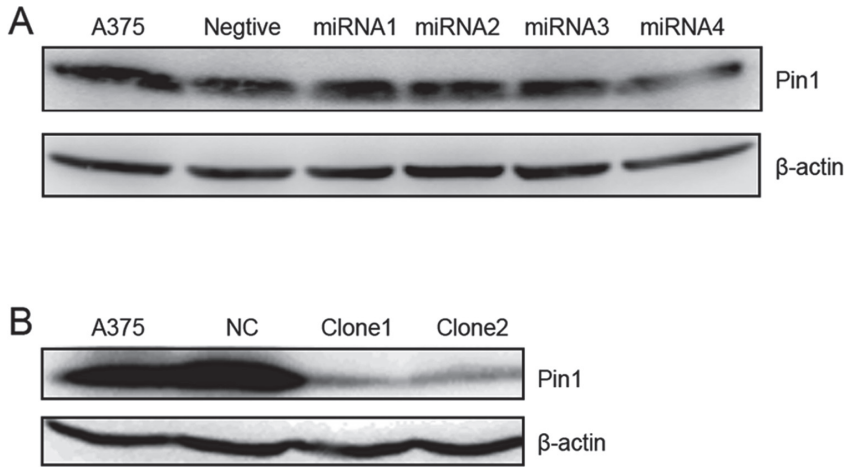

Figure 1. Western blot analysis of Pin1 expression in A375 cells, followed by miRNAi. (A) A375 cells were transiently transfected with four pcDNA6.2-GW/EmGFP-miR-Pin1 plasmids that targeted Pin1 with four different miRNA oligonucleotides, as well as the negative control plasmid. Seventy-two hours later, cells were lysed and subjected to western blot analysis with either anti-Pin 1 or anti- $\beta$-actin antibodies. The inhibitory percentages of four plasmids were $0 \%, 15 \%, 31 \%$ and $54 \%$, compared with negative control plasmid. (B) A375 cells were stably transfected with the most efficient plasmid, pcDNA6.2-GW/EmGFP-miR-Pin1 with miRNA4 oligonucleotides and the negative control. Western blot analysis of Pin1 and $\beta$-actin are shown in parental A375, NC, Clone1 and Clone 2 cells, in which Pin1 expression was significantly inhibited in Clone1 and Clone2, with the percentage of inhibition of $84.9 \%$ and $83.9 \%$ compared with NC, respectively $(\mathbf{P}<0.001)$.

plasmid. Briefly, A375 cells were seeded at a density of $6 \times 10^{5}$ cell/well in six-well tissue culture plates 1 day before transfection in order to achieve $80-90 \%$ confluency. Transfection was performed using Lipofectamine 2000 (Invitrogen) according to the manufacturer's protocol. At $24 \mathrm{hr}$ after transfection, the cells were passaged at 1:15 into fresh growth medium containing Blastidin (Invitrogen) at $7 \mu \mathrm{g} / \mathrm{ml}$ for clonal selection. Selection was maintained in a medium containing Blastidin at $3.5 \mu \mathrm{g} / \mathrm{ml}$ to ensure stable transfection.

MTS assay. Cells $\left(1 \times 10^{3}\right)$ were seeded into 96-well plates with complete medium. According to the manufacturer's protocol, assays were performed by adding $10 \mu \mathrm{L}$ MTS reagent (Promega, Madison, USA) directly into culture wells, incubating for $4 \mathrm{hr}$ and then recording the absorbance at $490 \mathrm{~nm}$ with a 96 -well plate reader every $24 \mathrm{hr}$ for 5 days. All experiments were performed in triplicate.

Colony formation assay. A total of 200 cells were plated in six-well plates, which yielded 150-200 colonies per well after approximately 10 days of culture. The growth of the colonies was examined 10 days later after crystal violet staining. Colonies that consisted of more than 50 cells were scored. The number of colonies was expressed as the mean and standard deviation (SD) of triplicate experiments.

Flow cytometry analysis. Cells $\left(1 \times 10^{6}\right)$ kept in culture with $10 \%$ serum were trypsinized, washed three times with phosphate-buffered saline (PBS), and fixed with cold 70\% ethanol for $24 \mathrm{hr}$ at $-20^{\circ} \mathrm{C}$. Before testing, the fixed cells were washed with PBS and re-suspended in PBS that contained $50 \mathrm{mg} / \mathrm{ml}$ propidium iodide and $0.5 \mathrm{mg} / \mathrm{ml}$ RNase 
A
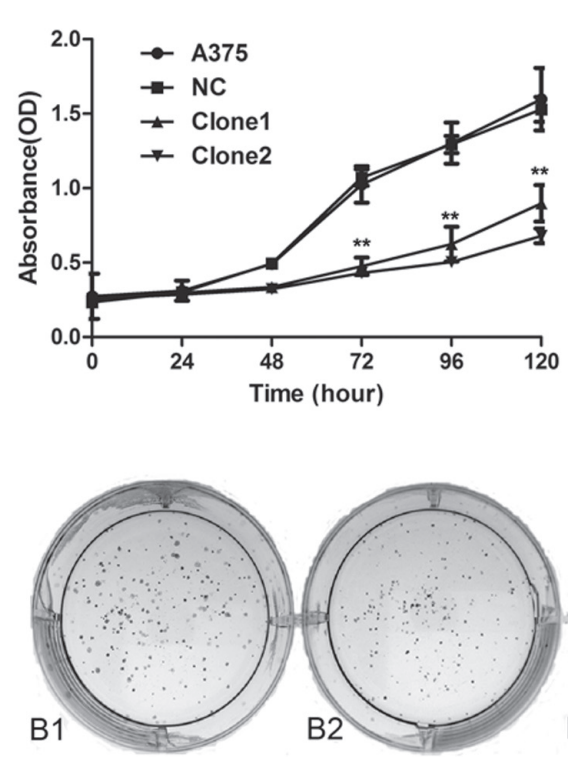

B
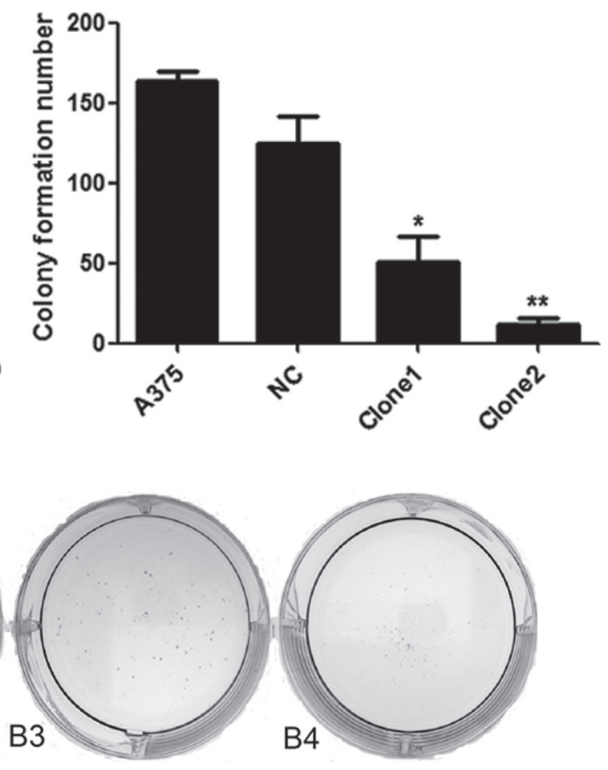

Figure 2. Pin 1 inhibition decreased proliferation and colony formation in A375 cells.

(A) Growth curves of melanoma cells (A375, NC, Clone1 and Clone2) by MTS assay. The data at each time point were derived from three independent experiments and the error bars represent the SD. The inhibitory rate of Clone1 and Clone2 was $55.5 \%$ and $59.8 \%$ for $72 \mathrm{~h}(\mathrm{P}<0.01), 51.8 \%$ and $61.0 \%$ for $96 \mathrm{~h}(\mathrm{P}<0.01), 41.2 \%$ and 55.5\% for $120 \mathrm{~h}(\mathrm{P}<0.01)$, respectively. $(\mathrm{N}=3)(\mathrm{B})$ Equal numbers of melanoma cells were seeded in six-well plates. After 10 days, the cells were fixed and stained with crystal violet and the number of colonies was counted from three independent experiments. $(\mathrm{N}=3) \mathrm{B} 1$ : A375; B2: NC; B3: Clone1; B4: Clone2.

A for $30 \mathrm{~min}$ at $37^{\circ} \mathrm{C}$. Cell cycle distribution was measured subsequently.

Western blot analysis. Total cellular protein was extracted using lysis buffer. Protein concentration was measured by the Bio-Rad protein assay. An equal amount of protein was separated using $10 \%$ and $12 \%$ SDS-PAGE and transferred to nitrocellulose membranes (Amersham, Bucks, UK). The membranes were blocked with 5\% skimmed milk in PBS and incubated overnight with primary antibodies, followed by horseradish-peroxidase-conjugated antibodies at room temperature. $\beta$-Actin was used as internal positive control. Primary antibodies included Pin1 (Santa Cruz Biotechnology, Santa Cruz, CA, USA), pAKT (Cell Signaling Technology, Danvers, MA, USA), AKT (Cell Signaling Technology), C-Jun $\mathrm{N}$-terminal kinase (JNK; Cell Signaling Technology), matrix metalloproteinase (MMP)-2 (Santa Cruz Biotechnology), $\beta$ catenin (Santa Cruz Biotechnology) and $\beta$-actin (Santa Cruz Biotechnology). Signals were visualized using an enhanced chemiluminescence system (Amersham).

Transwell migration assay. Transwell migration assay was performed by the Boyden chamber system (Neuro Probe, Gaithersburg, MD, USA) with a fibronectin-precoated (0.5 $\mathrm{mg} / \mathrm{ml}$ ) polycarbonate membrane $(8 \mu \mathrm{m}$ pore size). The lighter side of the polycarbonate membrane was precoated with 250 $\mu \mathrm{g} / \mathrm{ml}$ Matrigel (BD Labware, Franklin Lakes, NJ, USA). The bottom chambers were filled with $30 \mu \mathrm{l}$ RPMI 1640 serum-free medium that contained $2 \%$ bovine serum albumin (BSA), and the top chambers were filled with $50 \mu \mathrm{l}$ RPMI 1640 serum-free medium that contained $0.2 \%$ BSA. Cells $\left(1 \times 10^{5} /\right.$ well $)$ were added to the top chamber, followed by $16 \mathrm{hr}$ incubation at $37^{\circ} \mathrm{C}$ in a $5 \% \mathrm{CO}_{2}$ incubator. Three independent experiments were performed in triplicate. The cells were fixed in methanol and stained with hematoxylin. The top surface of the membrane was gently scrubbed with a cotton bud. The cells that had migrated to the lower side of the membrane were counted under a microscope (OLYMPUS IX70; Tokyo, Japan), and the percentage of migration were calculated.

Tumor formation in nude mice. Six to eight-weekold male nude mice $(B A L B / c-n u)$ were obtained from the Vital River Laboratory Animal Technology Co. Ltd (Beijing, China). All animals in our study were housed under pathogen-free conditions and maintained according to the guidelines of the Committee on Animals of the Institute of Materia Medica, Chinese Academy of Medical Sciences \& Peking Union Medical College. Three groups of cells (NC, Clone1 and Clone2) were washed and resuspended with normal saline. The cell suspensions $\left(1 \times 10^{7}\right.$ cells $)$ were injected subcutaneously into the BALB/c-nu mice. One week later, tumor formation was monitored and recorded every 3 or 4 days. Tumor volume was calculated by the formula: $a^{2} b / 2$, where $a$ and $b$ are the two maximum diameters measured by a sliding caliper. The mice were sacrificed after 21 days and 
A
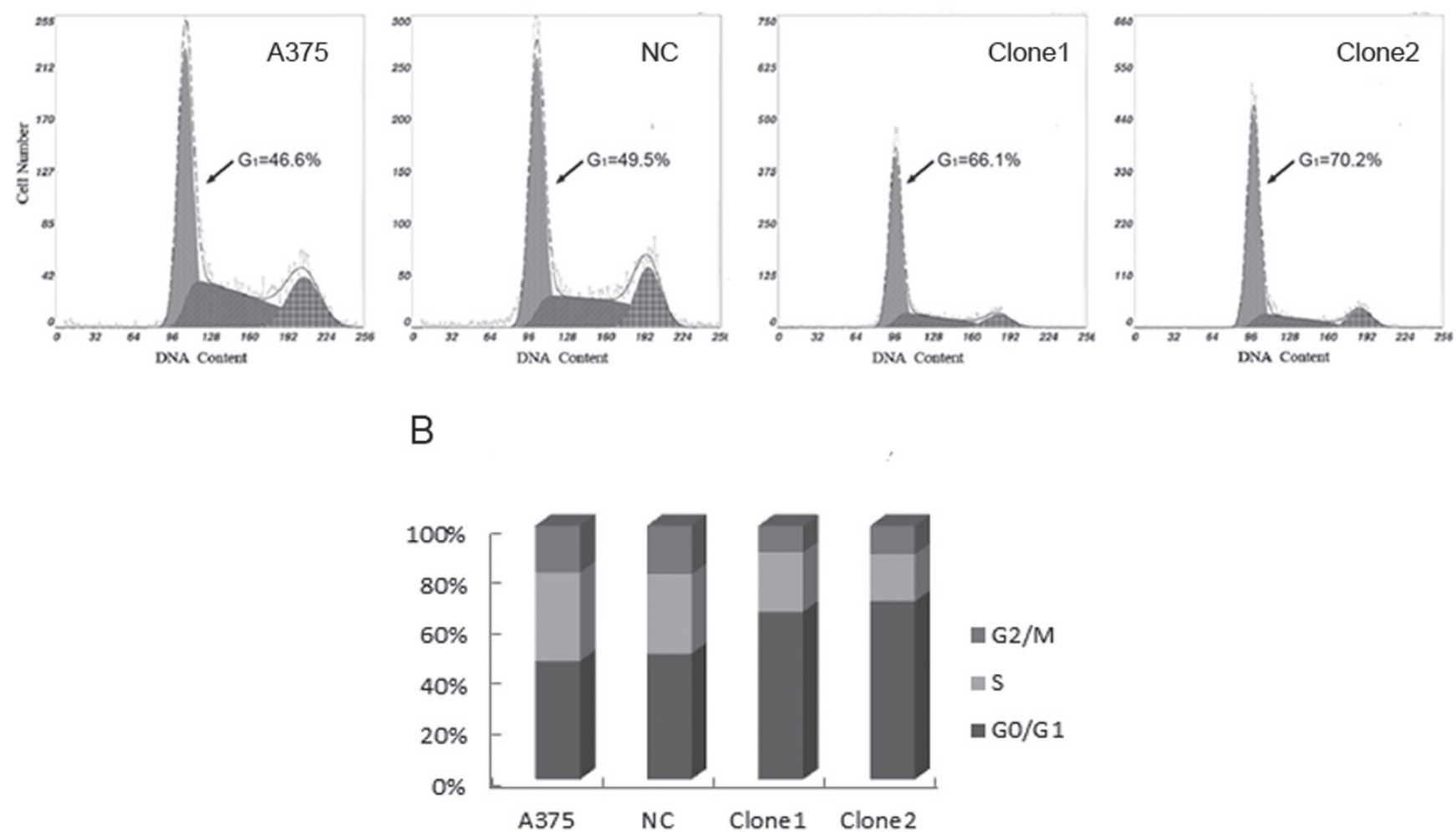

Figure 3. Flow cytometric analysis. (A) Cell cycle distribution of melanoma cells (A375, NC, Clone1 and Clone2) was detected by flow cytometric analysis. An increased proportion of cells at $G_{0} / G_{1}$ phase was found in Pin1-silenced clones. (B) The statistical graph of cell cycle distribution.

the tumors were weighed. A total of four nude mice were used for each group.

Statistical analysis. All experiments were repeated at least three times. One way ANOVA was used to evaluate the differences between experimental and control groups with SPSS version 13.0 (SPSS, Chicago, IL, USA).

\section{Results}

Inhibition of Pin1 expression by RNAi in A375 cells. To examine the role of Pin 1 in carcinogenesis of malignant melanoma, we depleted Pin 1 using the RNAi method in human malignant melanoma A375 cells, which have the advantage of high-proliferative and invasive activity that makes experimental protocols possible in vitro and in vivo. Four pcDNA6.2-GW/EmGFP-miR-Pin1 plasmids against Pin1 with four different synthesized miRNA oligonucleotides were constructed. Western blot analysis of transient transfection revealed that the pcDNA6.2-GW/EmGFP-miR-Pin1 plasmid with miRNA4 oligonucleotides showed the strongest inhibitory activity against Pin1 expression, whereas the pcDNA6.2-GW/EmGFP-miR-Negative plasmid showed no effect (Fig. 1). Subsequently, stable transfection using the
pcDNA6.2-GW/EmGFP-miR-Pin1 plasmid with miRNA4 oligonucleotides was performed and two single clones designated Clone1 and Clone2 were established. The A375 cells stably transfected with pcDNA6.2-GW/EmGFP-miR-Negative control plasmid were designated NC. As shown in Figure 1B, expression of Pin 1 was significantly suppressed in Clone1 and Clone2 $(84.9 \%$ and $83.9 \%$ inhibition respectively, analyzed by Image Pro, $\mathrm{P}<0.001$ ), but unchanged in NC compared with the parental cells.

Pin1 suppression retards proliferation of A375 cells. To investigate whether inhibition of Pin 1 expression can inhibit cell proliferation, we recorded cell growth curves by MTS assay. As shown in (Fig.2A), Pin1 inhibition resulted in a significant decrease in cell proliferation, whereas the NC grew normally as parental $\mathrm{A} 375$ cells. Compared with NC, the inhibitory rate of Clone1 and Clone2 were $55.5 \%$ and $59.8 \%$ for 72 hour (P $<0.01), 51.8 \%$ and $61.0 \%$ for 96 hour $(\mathrm{P}<0.01), 41.2 \%$ and $55.5 \%$ for 120 hour $(\mathrm{P}<0.01)$, respectively.

Pin1 suppression abrogates anchorage-dependent colony formation in $\mathbf{A} \mathbf{3 7 5}$ cells. To examine the effect of Pin1 inhibition on tumorigenic properties in A375 cells in vitro, we tested the anchorage-dependent colony formation ability of different cells. As shown in (Fig.2B), compared with the NC 
A

B
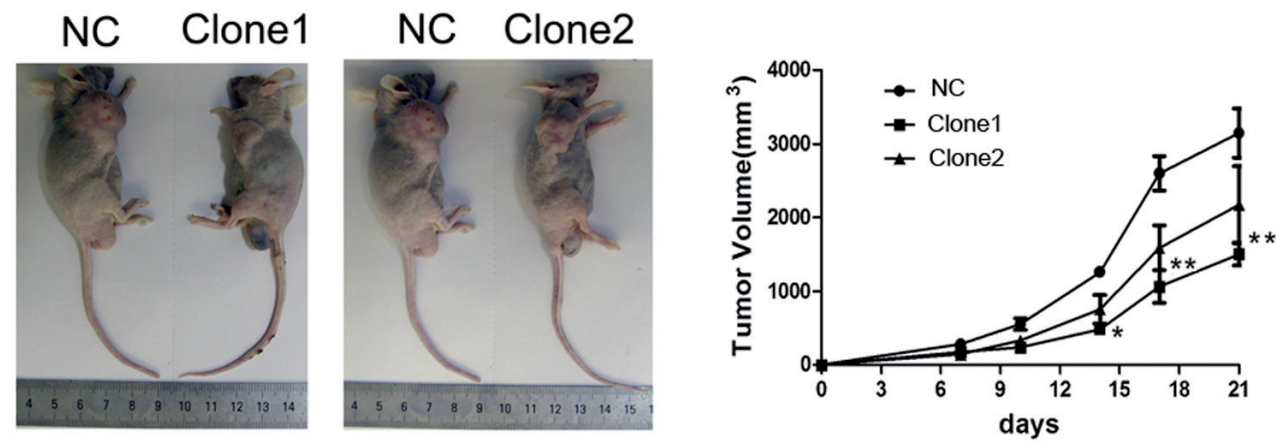

C
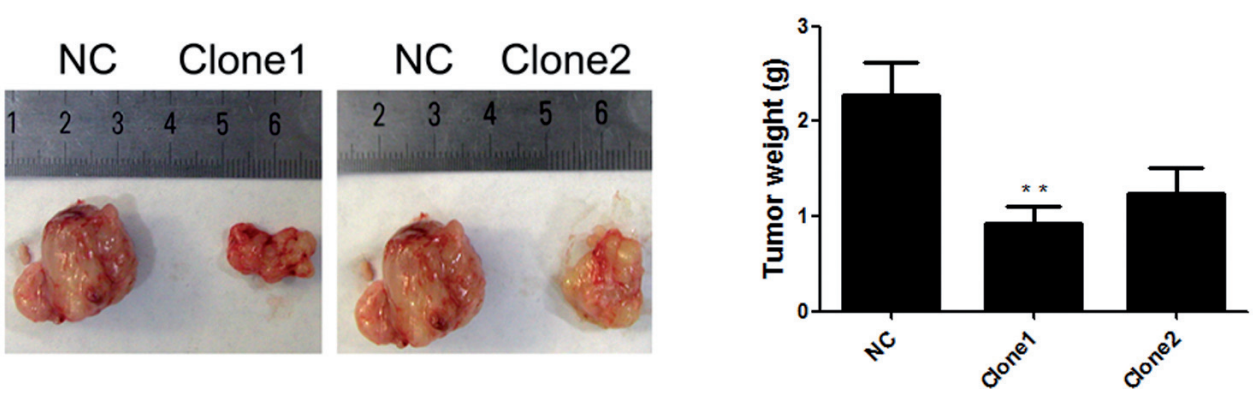

Figure 4. Pin1 inhibition decreased tumorigenicity in nude mice. (A) Melanoma cells (NC, Clone1 and Clone2) were inoculated subcutaneously into nude mice $(n=4)$. Representative tumor formation at 21 days after injection. (B) Tumor growth was monitored and tumor volumes were calculated. Compared with NC, the percentage of decrease of tumor volumes of Clone1 and Clone2 were $52.2 \%(P<0.01)$ and $30.8 \%(P>0.05)$ at day 21 after injection. $(C)$ Representative appearance of tumor mass resected from nude mice at 21 days after injection. (D) Mean tumor weight for four animals at 21 days after injection. Compared with NC, the percentage of decrease of tumor weight of Clone1 and Clone2 were $59.2 \%(P<0.01)$ and $45.5 \%(P>0.05)$, respectively.

group, colony formation of Clone1 and Clone2 was reduced by $59.0 \%(\mathrm{P}<0.05)$ and $90.3 \%(\mathrm{P}<0.01)$, respectively. In contrast, there was no significant difference between $\mathrm{NC}$ and parental A375 cells $(\mathrm{P}>0.05)$.

Pin1 suppression induces G0/G1 arrest. We determined whether loss of Pin1 expression affected cell cycle progression. As shown in (Fig.3), the proportion of cells residing in the G0/G1 phase of parental A375 and NC cells was $46.6 \%$ and $49.5 \%$, respectively. However, this proportion was significantly increased to $66.1 \%$ and $70.2 \%$ in Clone1 and Clone2, respectively, which indicated that depletion of Pin1 resulted in arrest in G0/G1 phase.

Pin1 suppression inhibits proliferation of A375 in vivo. The results presented above suggest that Pin 1 plays a dominant role in the proliferation of A357 cells in vitro. To investigate whether Pin 1 suppression could decrease tumorigenesis in vivo, xenograft studies were carried out using NC, Clonel and Clone2. As shown in (Fig.4), Clone1 and Clone2 with silenced Pin 1 expression showed an obvious decrease in tumor size (52.2\% and 30.8\% decrease respectively) and weight (59.2\% and $45.5 \%$ decrease respectively) at day 21 after subcutaneous inoculation, especially Clone1, compared with NC cells $(\mathrm{P}<0.01)$.
Pin1 suppression inhibits the invasive ability of A375 cells. To evaluate further the role of Pin1 in cell invasion, we performed an invasion assay to investigate any difference between these cells. As shown in (Fig.5), Clone1 and Clone2 showed a dramatic decrease in migration, in which the percentage of invaded cells was $45.2 \%(\mathrm{P}<0.05)$ and $27.4 \%(\mathrm{P}$ $<0.01$ ), respectively, compared with A375 cells. These results suggested that Pin 1 depletion could significantly suppress invasion of A375 cells.

Effects of Pin 1 suppression on related protein expression. It has been reported that Pin 1 regulates a series of target proteins, many of which are often deregulated during the course of oncogenesis. [19,20] To identify which proteins are inhibited by Pin 1 suppression in malignant melanoma, phosphorylation of Akt, and expression of JNK, MMP2 and $\beta$-catenin were measured by western blotting. Compared with NC, Clone1 and Clone2 showed a significant decrease in activated Akt (shown as pAkt-Ser473/Akt) (42.3\% and $46.2 \%$ down-regulation, $\mathrm{P}<0.05$ ), JNK2/3 (59.6\% and $76.6 \%$ down-regulation, $\mathrm{P}<0.01)$, JNK1 (60.8\% and 55.4\% down-regulation, $\mathrm{P}<0.01$ ) and pro-MMP2 (59.6\% and 76.6\% down-regulation, $\mathrm{P}<0.01$ ), whereas there was no conspicuous change in the expression of $\beta$-catenin (Fig. 6). 

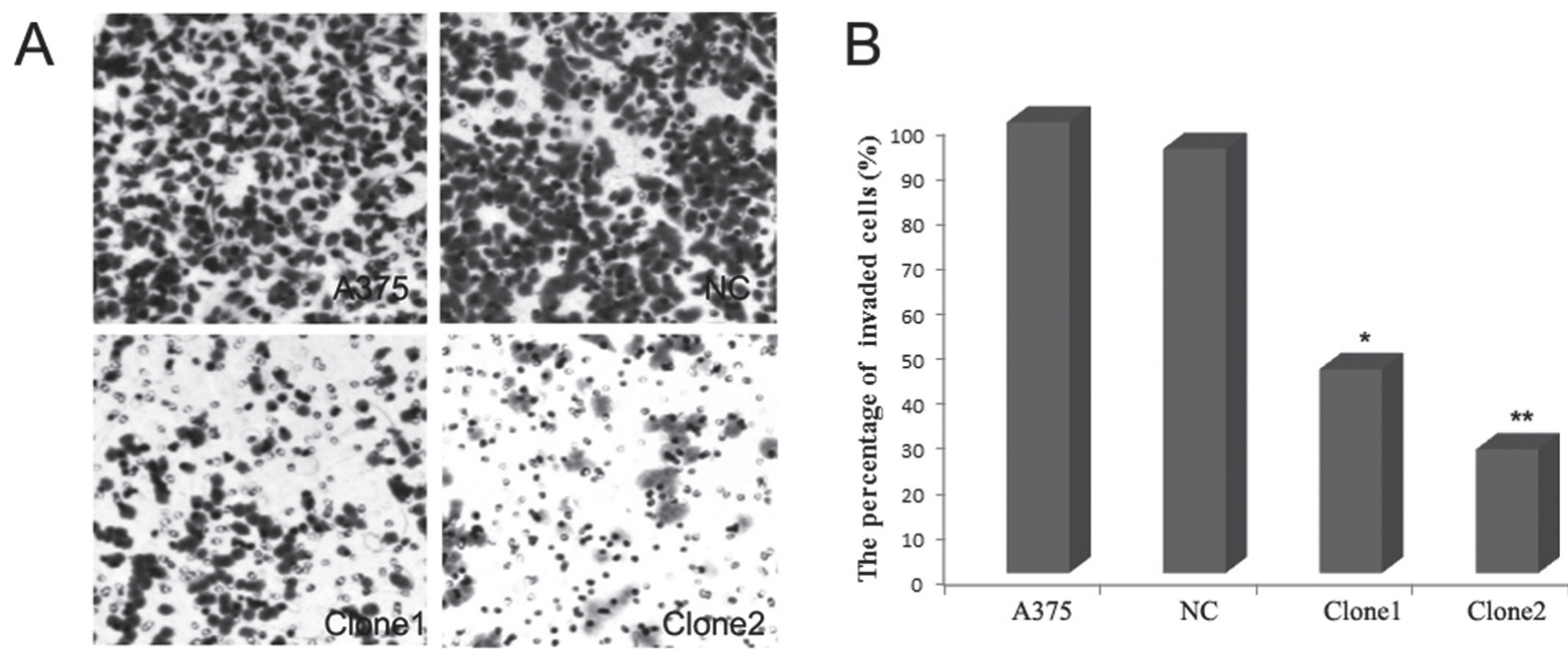

Figure 5. Depletion of Pin1 suppressed cell invasion. ( $N=3$ ) (A) Invasion of melanoma cells (A375, NC, Clone1 and Clone2) through reconstituted basement membrane (original magnification, 40×). (B) The percentage of invaded cells in the transwell migration assay.

\section{Discussion}

Although much research has indicated that Pin1 is markedly overexpressed in many different human cancers, including melanoma, it is still unclear whether Pin1 is involved in the carcinogenesis of malignant melanoma, and whether Pin1 suppression blocks tumorigenesis of malignant melanoma.

In the present study, we inhibited Pin 1 expression in A375 cells with miRNA plasmid and investigate whether Pin1 suppression could reverse the malignant phenotype. The cells of Clone1 and Clone2 with silenced Pin1 showed retarded proliferation and decreased ability of colony formation. The xenografted tumors from the cells of Clone1 and Clone2 were observed decreased in size and mass. These observations demonstrated the crucial role of Pin 1 in the regulation of the proliferation of melanoma. Further, we investigated whether this growth inhibition caused by Pin1 depletion was related to changes in cell cycle distribution. Flow cytometry analysis showed that both clones with silenced Pin1 expression had an increased proportion of cells residing in the G0/G1 phase, which could partially elucidate the growth inhibition phenomenon, and indicated that Pin 1 was mainly involved in the transition between G0/G1 and S phase in malignant melanoma A375 cells.

Malignant melanoma, one of the most malignant cutaneous tumors, is characterized by its high potential for invasiveness and metastasis. It is these characteristics that lead to extremely poor prognosis and high mortality.[4, 21,22] As shown in the present study, Pin 1 suppression greatly inhibited the invasive ability of A375 cells.

$\mathrm{AKT} /$ protein kinase B is a cardinal node in many signal cascades of receptor tyrosine kinase downstream pathways, and plays a pivotal role in diverse cellular functions such as

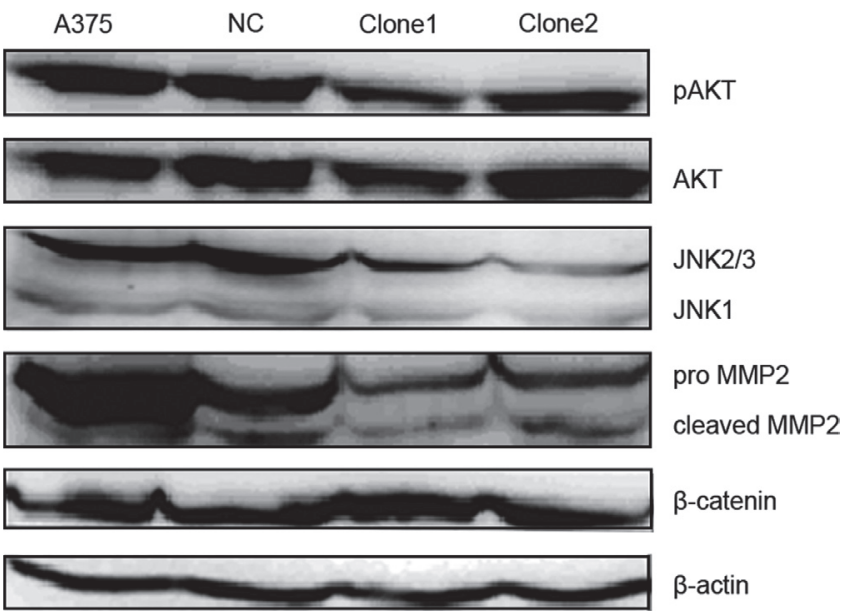

Figure 6. Pin1 inhibition induced downregulation of the phosphorylation of Akt $(P<0.05)$, the expression of JNK $(P<0.01)$ and pro-MMP2 (P $<0.01$ ), whose expression was associated with development of melanoma. Western blot analysis with antibodies specific for p-AKT, AKT, JNK, MMP2 and $\beta$-catenin is shown. $\beta$-actin levels are shown as an internal control. $(\mathrm{N}=3)$

proliferation, differentiation and migration[23-25]. Numerous studies have testified that AKT is frequently aberrantly regulated in a wide variety of human cancers including malignant melanoma, and increased phosphorylation of AKT in malignant melanoma is associated with tumor progression and shorter survival. Importantly, AKT has been recognized as a potential target in therapy of malignant melanoma[5, 2629]. In a study of breast cancer, Liao et al. [30] have found that the expression level of AKT phosphorylation at S473 is correlated with the level of Pin1, and their combined expression levels predict poorer prognosis than does either one alone. 
They have also shown that Pin1 can regulate AKT stability and phosphorylation at S473 through the phosphorylated Thr-Pro motifs of AKT.[30] Similarly, our study showed that Pin1 suppression inhibited phosphorylation of AKT at S473 in A375 cells. Thus, specific Pin1 depression could be an appealing strategy to inhibit deregulation of AKT in malignant melanoma.

JNKs, also referred to as stress-activated kinases, are an evolutionarily conserved sub-group of mitogen-activated protein kinases and are implicated in oncogenic transformation.[3134] Although its role in tumor development remains controversial, several studies have suggested that JNK is essential to the survival of melanoma, and JNK inhibition can suppress melanoma cell proliferation in vitro and in vivo. [35, 36] In a study of Alzheimer's disease, Rudrabhatla et al.[37] have reported that Pin1 can regulate the activity of JNK3, a JNK isoform that is restricted to brain, heart and testes.[38, 39] However, we do not know whether Pin1 is involved in regulation of JNKs in cancer. In the present study, we found for the first time that Pin1 inhibition suppressed JNK expression dramatically. Although further detailed analysis, such as the exact mechanism of direct JNK inhibition, is necessary, our results indicated that Pin 1 can affect JNK expression in malignant melanoma, and the growth inhibition of malignant melanoma that is mediated by Pin 1 suppression might be related to JNK inhibition.

It is well established that upregulation of MMPs in melanoma is one of the most crucial factors that lead to tumor invasion and metastasis.[40-43] In particular, patients with high expression of MMP2 often have worse prognosis.[40, 44] In fact, Ryo et al. have found that inhibition of Pin1 suppresses expression of MMP2 in prostate cancer cells. [45] Our study showed that, in melanoma A375 cells, Pin 1 inhibition decreased expression of pro-MMP2 greatly. Although the cleaved MMP2 did not change significantly, there was a tendency to decline. Further research is under way in our laboratory to investigate this phenomenon.

$\beta$-catenin is a key component of the Wnt signaling pathway, and is closely associated with tumor cell proliferation and invasion.[46] However, its exact role in melanoma remains controversial. $[47,48] \beta$-catenin is also one of the most important substrates regulated by Pin1.[12, 13, 49, 50] However, in our study, we did not find any changes in the expression of $\beta$-catenin followed by Pin1 interference. The fact that Pin 1 did not affect expression of $\beta$-catenin in A375 cells indicated that the relationship between Pin 1 and $\beta$-catenin could have tumor origin specificity.

In summary, Pin 1 suppresion by miRNAi prevented human melanoma A375 cell proliferation and invasion in vitro and tumor growth in vivo, that was accompanied with the inhibition of a variety of tumorigenesis-related oncoproteins. These findings indicate that Pin 1 might be a potential molecular target for malignant melanoma therapy. Our further studies are going to be focused on the mechanism of Pin1 regulating proliferation, apoptosis and angiogenesis .
Acknowledgments: This work is supported by the National Natural Science Foundation of China (Grant No. 30500634) and the National Science and Technology Major Project of the Ministry of Science and Technology of China (Grant No. 2009ZX09301-003-9-1).

\section{References}

[1] GIBLIN AV, THOMAS JM. Incidence, mortality and survival in cutaneous melanoma. J Plast Reconstr Aesthet Surg 2007; 60: 32-40. http://dx.doi.org/10.1016/j.bjps.2006.05.008

[2] CHO YR, CHIANG MP. Epidemiology, staging (new system), and prognosis of cutaneous melanoma. Clin Plast Surg 2010; 37: 47-53. http://dx.doi.org/10.1016/j.cps.2009.07.001

[3] RUBIN KM, LAWRENCE DP. Your patient with melanoma: staging, prognosis, and treatment. Oncology (Williston Park) 2009; 23: 13-21.

[4] MARKOVIC SN, ERICKSON LA, FLOTTE TJ, KOTTSCHADE LA, MCWILLIAMS RR, et al. Metastatic malignant melanoma. G Ital Dermatol Venereol 2009; 144: $1-26$.

[5] AMARAVADI RK, FLAHERTY KT. Targeted therapy for metastatic melanoma. Clin Adv Hematol Oncol 2007; 5: 386-394.

[6] JILAVEANU LB, AZIZ SA, KLUGER HM. Chemotherapy and biologic therapies for melanoma: do they work? Clin Dermatol 2009; 27: 614-625. http://dx.doi.org/10.1016/ j.clindermatol.2008.09.020

[7] BLUME-JENSEN P, HUNTER T. Oncogenic kinase signalling. Nature 2001; 411: 355-365. http://dx.doi.org/ $\underline{10.1038 / 35077225}$

[8] LU KP, HANES SD, HUNTER T. A human peptidyl-prolyl isomerase essential for regulation of mitosis. Nature 1996; 380: 544-547. http://dx.doi.org/10.1038/380544a0

[9] LU KP, LIOU YC, ZHOU XZ. Pinning down proline-directed phosphorylation signaling. Trends Cell Biol 2002; 12: 164-172. http://dx.doi.org/10.1016/S0962-8924(02)02253-5

[10] BAO L, KIMZEY A, SAUTER G, SOWADSKI JM, LU KP. Prevalent overexpression of prolyl isomerase Pin 1 in human cancers. Am J Pathol 2004; 164: 1727-37. http://dx.doi. org/10.1016/S0002-9440(10)63731-5

[11] WULF GM, RYO A, WULF GG, LEE SW, NIU T, et al. Pin1 is overexpressed in breast cancer and cooperates with Ras signaling in increasing the transcriptional activity of c-Jun towards cyclin D1. Embo J 2001; 20: 3459-3472. http://dx.doi. org/10.1093/emboj/20.13.3459

[12] PANG R, YUEN J, YUEN MF, LAI CL, LEE TK, et al. PIN1 overexpression and beta-catenin gene mutations are distinct oncogenic events in human hepatocellular carcinoma. Oncogene 2004; 23: 4182-4186. http://dx.doi.org/10.1038/ sj.onc. 1207493

[13] KIM CJ, CHO YG, PARK YG, NAM SW, KIM SY, et al. Pin1 overexpression in colorectal cancer and its correlation with aberrant beta-catenin expression. World J Gastroenterol 2005; 11: 5006-5009.

[14] HE J, ZHOU F, SHAO K, HANG J, WANG H, et al. Overexpression of Pin 1 in non-small cell lung cancer (NSCLC) and its correlation with lymph node metastases. 
Lung Cancer 2007; 56: 51-58. http://dx.doi.org/10.1016/ j.lungcan.2006.11.024

[15] MIYASHITA H, MORI S, MOTEGI K, FUKUMOTO M, UCHIDA T. Pin 1 is overexpressed in oral squamous cell carcinoma and its levels correlate with cyclin D1 overexpression. Oncol Rep 2003; 10: 455-461.

[16] AYALA G, WANG D, WULF G, FROLOV A, LI R, et al. The prolyl isomerase Pin1 is a novel prognostic marker in human prostate cancer. Cancer Res 2003; 63: 6244-6251.

[17] FUKUCHI M, FUKAI Y, KIMURA H, SOHDA M, MIYAZAKI T, et al. Prolyl isomerase Pin1 expression predicts prognosis in patients with esophageal squamous cell carcinoma and correlates with cyclinD1 expression. Int J Oncol 2006; 29: 329-334.

[18] LU KP. Prolyl isomerase Pin1 as a molecular target for cancer diagnostics and therapeutics. Cancer Cell 2003; 4: 175-180. http://dx.doi.org/10.1016/S1535-6108(03)00218-6

[19] RYO A, LIOU YC, LU KP, WULF G. Prolyl isomerase Pin1: a catalyst for oncogenesis and a potential therapeutic target in cancer. J Cell Sci 2003; 116: 773-83. http://dx.doi.org/10.1242/ jcs.00276

[20] YEH ES, MEANS AR. PIN1, the cell cycle and cancer. Nat Rev Cancer 2007; 7: 381-388. http://dx.doi.org/10.1038/nrc2107

[21] ESSNER R. Surgical treatment of malignant melanoma. Surg Clin North Am 2003; 83: 109-156. http://dx.doi.org/10.1016/ S0039-6109(02)00205-0

[22] KASHANI-SABET M, SAGEBIEL RW, FERREIRA CM, NOSRATI M, MILLER JR 3rd. Vascular involvement in the prognosis of primary cutaneous melanoma. Arch Dermatol 2001; 137: 1169-1173.

[23] TESTA JR, TSICHLIS PN. AKT signaling in normal and malignant cells. Oncogene 2005; 24: 73917393.

[24] YANG ZZ, TSCHOPP O, BAUDRY A, DUMMLER B, HYNX D, et al. Physiological functions of protein kinase B/Akt. Biochem Soc Trans 2004; 32: 350-354. http://dx.doi. org/10.1042/BST0320350

[25] DUMMLER B, HEMMINGS BA. Physiological roles of PKB/ Akt isoforms in development and disease. Biochem Soc Trans 2007; 35: 231-235. http://dx.doi.org/10.1042/BST0350231

[26] STEELMAN LS, STADELMAN KM, CHAPPELL WH, HORN $\mathrm{S}$, BASECKE J, et al. Akt as a therapeutic target in cancer. Expert Opin Ther Targets 2008; 12: 1139-1165. http://dx.doi. org/10.1517/14728222.12.9.1139

[27] $\mathrm{CH}^{\prime} \mathrm{NG}$ S, TAN ST. Genetics, cellular biology and tumor microenvironment of melanoma. Front Biosci 2009; 14: 918-928.

[28] IBRAHIM N, HALUSKA FG. Molecular pathogenesis of cutaneous melanocytic neoplasms. Annu Rev Pathol 2009; 4: 551-579. http://dx.doi.org/10.1146/annurev. pathol.3.121806.151541

[29] MADHUNAPANTULA SV, ROBERTSON GP. The PTENAKT3 signaling cascade as a therapeutic target in melanoma. Pigment Cell Melanoma Res 2009; 22: 400-419. http://dx.doi. org/10.1111/j.1755-148X.2009.00585.X

[30] LIAO Y, WEI Y, ZHOU X, YANG JY, DAI C, et al. Peptidyl-prolyl cis/trans isomerase Pin 1 is critical for the regulation of $\mathrm{PKB} / \mathrm{Akt}$ stability and activation phospho- rylation. Oncogene 2009; 28: 2436-2445. http://dx.doi. org/10.1038/onc. 2009.98

[31] PAPACHRISTOU DJ, BATISTATOU A, SYKIOTIS GP, VARAKIS I, PAPAVASSILIOU AG. Activation of the JNK-AP-1 signal transduction pathway is associated with pathogenesis and progression of human osteosarcomas. Bone 2003; 32: 364-371. http://dx.doi.org/10.1016/S8756-3282(03)00026-7 [32] YANG YM, BOST F, CHARBONO W, DEAN N, MCKAY R, et al. C-Jun $\mathrm{NH}(2)$-terminal kinase mediates proliferation and tumor growth of human prostate carcinoma. Clin Cancer Res 2003; 9: 391-401.

[33] HESS P, PIHAN G, SAWYERS CL, FLAVELL RA, DAVIS RJ. Survival signaling mediated by c-Jun $\mathrm{NH}(2)$-terminal kinase in transformed B lymphoblasts. Nat Genet 2002; 32: 201-205. http://dx.doi.org/10.1038/ng946

[34] ZENZ R, WAGNER EF. Jun signalling in the epidermis: From developmental defects to psoriasis and skin tumors. Int J Biochem Cell Biol 2006; 38: 1043-1049. http://dx.doi. org/10.1016/j.biocel.2005.11.011

[35] GAO YJ, CHENG JK, ZENG Q, XU ZZ, DECOSTERD I, et al. Selective inhibition of JNK with a peptide inhibitor attenuates pain hypersensitivity and tumor growth in a mouse skin cancer pain model. Exp Neurol 2009; 219: 146-155. http://dx.doi. org/10.1016/j.expneurol.2009.05.006

[36] ALEXAKI VI, JAVELAUD D, MAUVIEL A. JNK supports survival in melanoma cells by controlling cell cycle arrest and apoptosis. Pigment Cell Melanoma Res 2008; 21: 429-438. http://dx.doi.org/10.1111/j.1755-148X.2008.00466.x

[37] RUDRABHATLA P, ZHENG YL, AMIN ND, KESAVAPANY S, ALBERS W. Pin1-dependent prolyl isomerization modulates the stress-induced phosphorylation of high molecular weight neurofilament protein. J Biol Chem 2008; 283: 26737-26747. http://dx.doi.org/10.1074/jbc.M801633200

[38] JOHNSON GL, NAKAMURA K. The c-jun kinase/stressactivated pathway: regulation, function and role in human disease. Biochim Biophys Acta 2007; 1773: 1341-1348.

[39] MANNING AM, DAVIS RJ. Targeting JNK for therapeutic benefit: from junk to gold? Nat Rev Drug Discov 2003; 2: 554-565. http://dx.doi.org/10.1038/nrd1132

[40] HOFMANN UB, WESTPHAL JR, VAN MUIJEN GN, RUITER DJ. Matrix metalloproteinases in human melanoma. J Invest Dermatol 2000; 115: 337-344.

[41] KERKELA E, SAARIALHO-KERE U. Matrix metalloproteinases in tumor progression: focus on basal and squamous cell skin cancer. Exp Dermatol 2003; 12: 109-125. http://dx.doi. org/10.1034/j.1600-0625.2003.120201.x

[42] SCHNAEKER EM, OSSIG R, LUDWIG T, DREIER R, OBERLEITHNER H, et al. Microtubule-dependent matrix metalloproteinase-2/matrix metalloproteinase-9 exocytosis: prerequisite in human melanoma cell invasion. Cancer Res 2004; 64: 8924-8931. http://dx.doi.org/10.1158/0008-5472. CAN-04-0324

[43] COTIGNOLA J, REVA B, MITRA N, ISHILL N, CHUAI S, et al. Matrix Metalloproteinase-9 (MMP-9) polymorphisms in patients with cutaneous malignant melanoma. BMC Med Genet 2007; 8: 10. http://dx.doi.org/10.1186/1471-2350-8$\underline{10}$ 
[44] RIA R, REALE A, CASTROVILLI A, MANGIALARDI G, DAMMACCO F, et al. Angiogenesis and progression in human melanoma. Dermatol Res Pract; 2010: 185687.

[45] RYO A, UEMURA H, ISHIGURO H, SAITOH T, YAMAGUCHI A, et al. Stable suppression of tumorigenicity by Pin1-targeted RNA interference in prostate cancer. Clin Cancer Res 2005; 11: 7523-7531. http://dx.doi.org/10.1158/ 1078-0432.CCR-05-0457

[46] MOON RT, KOHN AD, DE FERRARI GV, KAYKAS A. WNT and beta-catenin signalling: diseases and therapies. Nat Rev Genet 2004; 5: 691-701.

[47] WIDLUND HR, HORSTMANN MA, PRICE ER, CUI J, LESSNICK SL, et al. Beta-catenin-induced melanoma growth requires the downstream target Microphthalmia-associated transcription factor. J Cell Biol 2002; 158: 1079-1087. http:// dx.doi.org/10.1083/jcb.200202049
[48] CHIEN AJ, MOORE EC, LONSDORF AS, KULIKAUSKAS RM, ROTHBERG BG, et al. Activated Wnt/beta-catenin signaling in melanoma is associated with decreased proliferation in patient tumors and a murine melanoma model. Proc Natl Acad Sci U S A 2009; 106: 1193-1198. http://dx.doi. org/10.1073/pnas.0811902106

[49] RYO A, NAKAMURA M, WULF G, LIOU YC, LU KP. Pin1 regulates turnover and subcellular localization of beta-catenin by inhibiting its interaction with APC. Nat Cell Biol 2001; 3: 793-801. http://dx.doi.org/10.1038/ncb0901-793

[50] CHEN SY, WULF G, ZHOU XZ, RUBIN MA, LU KP, et al. Activation of beta-catenin signaling in prostate cancer by peptidyl-prolyl isomerase Pin1-mediated abrogation of the androgen receptor-beta-catenin interaction. Mol Cell Biol 2006; 26: 929-939. http://dx.doi.org/10.1128/MCB.26.3.929939.2006 